\title{
Animaux transgéniques: une voie nouvelle pour l'étude du développement
}

L'introduction de gènes étrangers dans l'œuf fécondé de souris constitue une technique récente extrêmement précieuse pour étudier l'expression des gènes au cours du développement et de la différenciation tissulaire.

\section{Charles Babinet}

Directeur de Recherche au Cnrs

Dominique Morello

Chargée de Recherche au Cnrs

\section{ADRESSE}

C. Babinet, D. Morello : Unité de génétique des mammiferes, Institut Pasteur, 25, rue du Docteur-Roux, 75724 Paris Cedex 15.

\section{TIRES A PART}

C. Babinet : Unité de génétique des mammiferes, Institut Pasteur, 25, rue du Docteur-Roux, 75724 Paris Cedex 15. 'un des problèmes fondamentaux posé à la biologie moderne est celui de l'élucidation des processus qui permettent à une cellule unique, l'œuf, de donner naissance à un organisme complexe comme celui par exemple d'une souris.

En dépit de notre ignorance quasi totale de ces processus, une conclusion s'est dégagée d'un ensemble de données obtenues par la biologie moléculaire depuis que l'ADN a été reconnu comme le dépositaire des caractères héréditaires : le développement d'un organisme résulte de l'expression différentielle et coordonnée dans le temps et dans l'espace de différents gènes ou groupes de gènes. Dès lors, la question du contrôle de l'expression des gènes devient l'une des questions-clés dans l'étude du développement.

Différentes stratégies ont été employées dans un passé récent pour tenter d'y répondre : elles reposent en particulier sur le transfert de gènes clonés dans des cellules de types variés maintenues en culture et sur l'étude de leur expression dans différentes conditions. Mais il est clair que l'étude in vitro a ses limites : d'une part, un grand nombre des types cellulaires constituant un organisme ne peut être maintenu en culture, d'autre part et surtout, l'étude du contrôle de l'expression de ces gènes au cours du développement est impossible dans ces cultures.

\section{Obtention de souris transgéniques}

Une autre stratégie [r], mise en œuvre récemment et maintenant en pleine expansion, a donc été employée : le gène est injecté au tout début du développement dans un pronucleus de l'œuf de souris tout juste fécondé (figure I, p. suivante). Dans un nombre de cas peu élevé mais tout de même significatif, le gène s'intègre avant la première division de l'œuf et va donc être transmis au même titre que l'ensemble des gènes de l'œuf hôte à toutes les cellules de l'organisme né de cet 


\section{RÉFÉRENCES}

I. Brinster RL, Palmiter RD. Introduction of genes into the germ line of animals. In: Riss $\mathrm{AL}$, ed. Harvey Lectures. New York: vol 80, 1986.

2. Gordon JW, Scangos GA, Plotkin DJ, Barbosa JA, Ruddle FK. Genetic transformation of mouse embryos by microinjection of purified DNA. Proc Natl Acad Sci USA 1980; 77: 7380-4.

3. Swift GH, Hammer RE, MacDonald RJ, Brinster RL. Tissue specific expression of the rat pancreatic elastase-I gene in transgenic mice. Cell 1984; 38: 639-46.

4. Ornitz DM, Palmiter RD, Hammer RE, Brinster RL, Swift GH, MacDonald RJ. Specific expression of clastase-human growth hormone fusion gene in pancreatic acinar cells of transgenic mice. Nature 1985; 313: 600-2.

5. Lacy E, Roberts S, Evans EP, Burtenshaw $\mathrm{MD}$, Costantini FD. A foreign $\beta$-globin gene in transgenic mice: integration at abnormal chromosomal positions and expression in inappropriate tissues. Cell 1983; 34: 343-58.

6. Palmiter RD, Norstedt G, Gelinas RE, Hammer RE, Brinster RL. Metallothionein-human $\mathrm{GH}$ fusion genes stimulate growth of mice. Science 1983; 222: 809-14.

7. Mougneau E, Glaichenhaus N, Cuzin F. Analyse génétique des étapes précoces de la progression tumorale: médecine/sciences $1985 ;$ I : 86-90.

8. Hanahan D. Heritable formation of pancreatic $\beta$-cell tumors in transgenic mice expressing recombinant insulin/simian virus 40 oncogenes. Nature 1985; 315: I1 5-22.

9. Brinster RL, Chen HY, Messing A, Van Dyke T, Levine A J, Palmiter RD. Transgenic mice harbouring $\mathrm{SV}_{40} \mathrm{~T}$-Antigen genes develop characteristic brain tumors. Cell 1984; 37: 367-79.

I0. Stewart TA, Pattengale PK, Leder P. Spontaneous mammary adenocarcinomas in transgenic mice that carry and express $\mathrm{MTV} /$ myc fusion genes. Cell I984; 38: 627-37.

II. Storb U, O'Brien RL, McMullen MD, Gollahon KA, Brinster RL. High expression of cloned immunoglobutin $K$ gene in transgenic mice is restricted to B lymphocytes. Nature 1984; 310 : 238-41.

12. Grosschedl R, Weaver D, Baltimore D, Costantini F. Introduction of a $\mu$ immunoglobulin gene into the mouse germ line: specific expression in lymphoid cells and synthesis of functional antibody. Cell 1984; 38: 647-58.

13. Rusconi S, Kohler G. Transmission and expression of a specific pair of rearranged immunoglobulin $\mu$ and $\kappa$ genes in a transgenic mouse line. Nature 1985; 314: 330-4.

14. Ritchie KA, Brinster RL, Storb U. Allelic exclusion and control of endogenous immunoglobulin gene rearrangement in transgenic mice. œuf. En principe, l'animal ainsi obtenu, appelé transgénique, constitue un système idéal pour l'étude de l'expression du gène en question : en effet, celui-ci a été soumis à tous les facteurs qui agissent normalement sur son expression au cours du développement. D'autre part il est possible de mesurer cette expression à tout moment et dans tout type cellulaire. $\mathrm{Si}$ le gène injecté est exprimé de manière correcte, c'està-dire au bon moment et dans les cellules appropriées, on peut alors envisager de répéter l'expérience avec des versions modifiées du gène : cela doit permettre à terme de découvrir les séquences d'ADN jouant un rôle dans l'expression spé- cifique d'un gène donné d'une part, et dans la mise en route de son expression au cours du développement d'autre part. Ajoutons enfin que, du fait de son intégration dans toutes les cellules de l'hôte et en particulier dans celles de la lignée germinale, le gène étranger est transmis aux générations suivantes : des lignées d'animaux transgéniques peuvent donc être maintenues.

La première démonstration de l'intégration stable d'ADN étranger dans le génome d'une souris via l'injection dans un pronucleus de l'œuf fécondé remonte à la fin de l'année I980 [2]. Depuis cette date, plusieurs gènes ont été injectés (et leur nombre ne cesse d'augmenter) et les

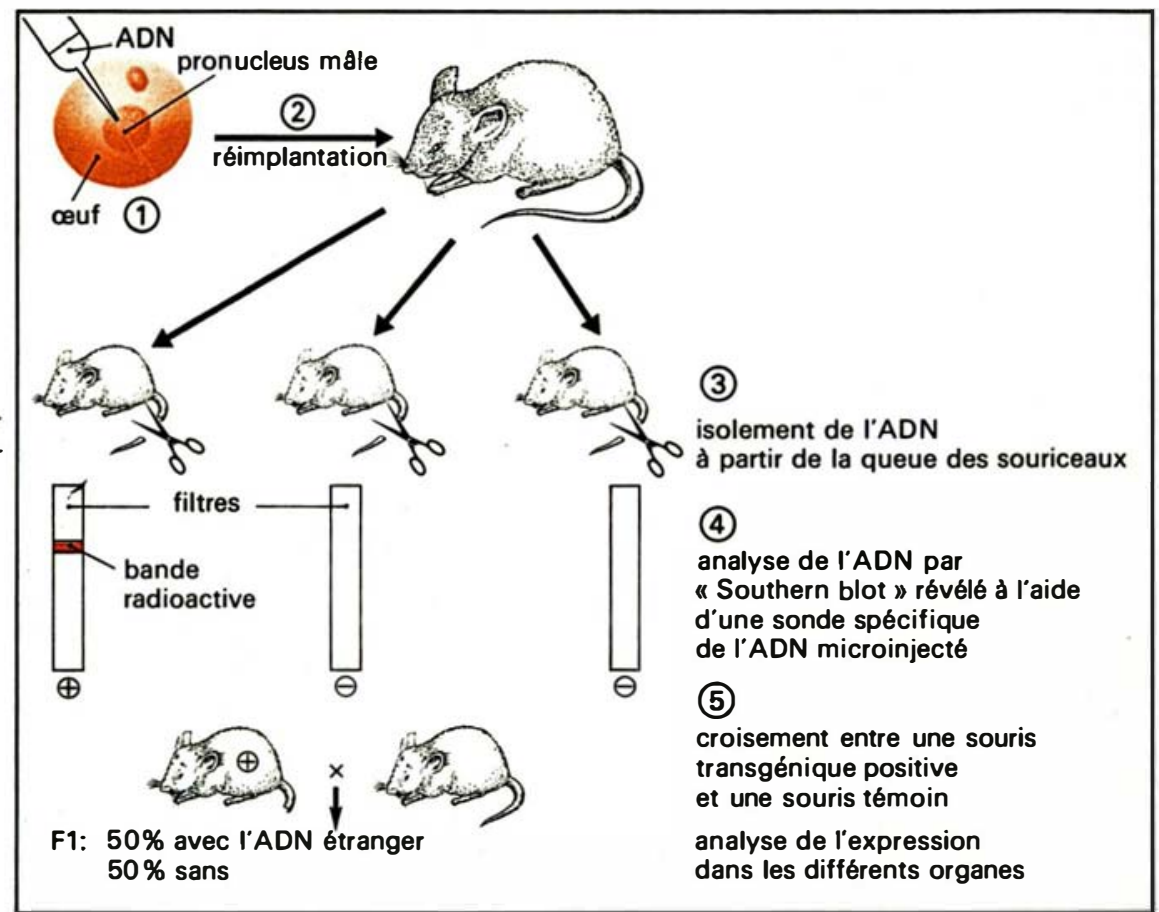

Figure 1. Schéma d'obtention et d'analyse des souris transgéniques.

(1) Quelques centaines de copies du gène étranger (ADN) sont injectées, à l'aide d'une micropipette dans le pronucleus d'un œuf fécondé.

(2) Les œufs ainsi traités sont réimplantés dans l'oviducte d'une mère "porteuse 11.

(3) Trois semaines après la naissance, de l'ADN est préparé à partir d'un petit bout de la queue des jeunes souris.

(4) L'ADN est analysé par Southern Blot (Lexique médecine/sciences $n^{\circ} 2$, vol. 2) à l'aide d'une sonde spécifique du gène étranger, ce qui permet de repérer les souris ayant intégré l'ADN injecté (souris transgéniques).

(5) Analyse des souris transgéniques. a) Expression du gène exogène dans les différents organes (ARN messager, protéine). b) Croisement avec des souris normales pour analyser la transmission du gène et éventuellement obtenir des lignées de souris transgéniques. 


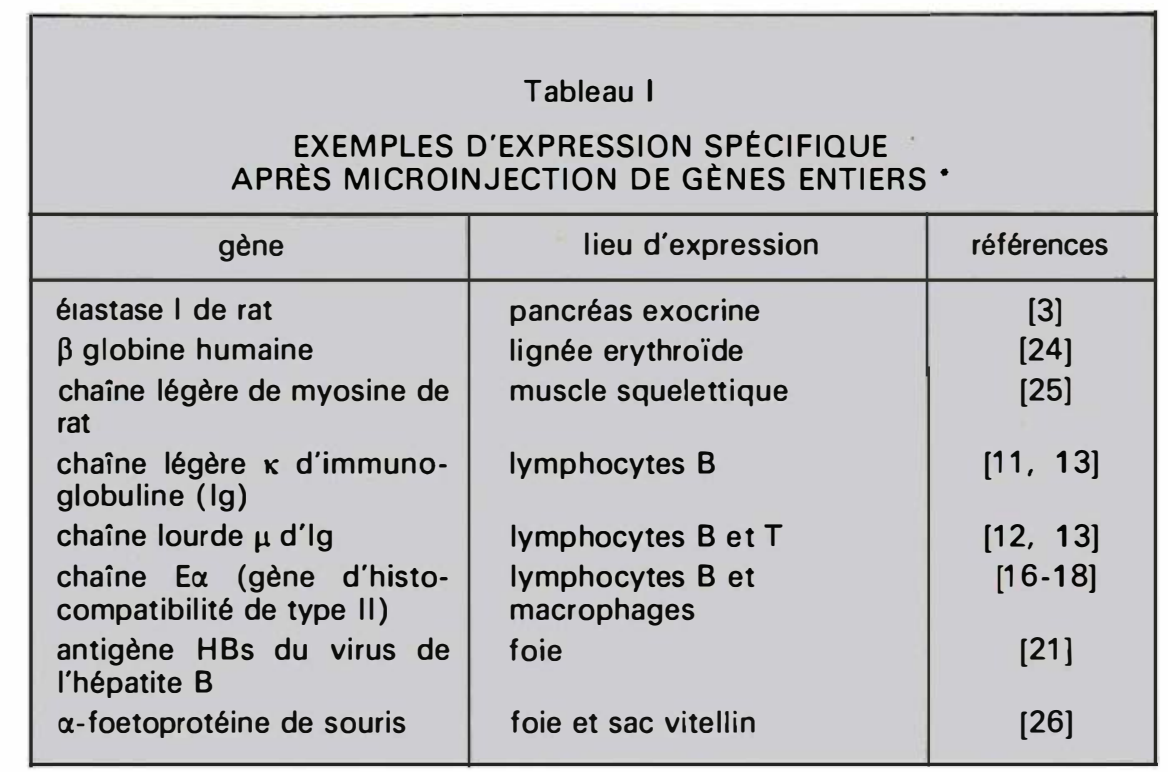

- Les séquences injectées comprennent les séquences codantes et les séquences adjacentes en $5^{\prime}$ et $3^{\prime} d^{\prime}$ un gène donné. au cours de ces études. D'une part, l'intégration de séquences étrangères dans l'ADN des souris transgéniques semble se faire en un seul site (parfois deux) et le nombre de séquences intégrées est variable (de I à parfois plus de I00); en outre, lors d'intégrations de plusieurs copies du gène, celles-ci sont très généralement disposées en tandem dans le sens tête-queue $\left(5^{\prime} \rightarrow 3^{\prime}\right.$, $\left.5^{\prime} \rightarrow 3^{\prime}\right)$. D'autre part, même s'il est trop tôt pour dire que l'intégration se fait au hasard, il est clair que de nombreux sites sont accessibles : d'une souris transgénique à l'autre, les sites sont toujours différents. Un corollaire à ces observations, et qui n'est pas sans conséquences, est que l'intégration des gènes exogènes ne se fait pas par recombinaison homologue avec le gène endogène. Enfin, l'analyse de l'ensemble des souris transgéniques obtenues dans ce type d'expérience a montré que le niveau d'expression des gènes exogènes pouvait être très variable, par comparaison avec l'expression du gène endogène. L'interprétation plausible de cette dernière observation est que l'environnement chromatinien du gène est important pour son fonctionnement correct. C'est ainsi que, si le gène s'intègre dans une région "silencieuse " du génome, cela peut entraîner son absence totale d'expression. A l'inverse, son intégration dans une région chromatinique très active pour l'expression dans un tissu donné peut avoir pour conséquence directe une expression erratique : c'est de cette manière qu'a été interprétée la présence de $\beta$-globine synthétisée à partir d'un gène exogène dans le tissu musculaire d'une souris transgénique [5]. L'ensemble de ces observations renforce l'idée que des interactions à distance et liées probablement à la structure globale de la chromatine sont également importantes dans l'expression appropriée des gènes. Grâce à l'étude des premières souris transgéniques, deux faits remarquables ont donc été établis : l'intégration de l'ADN exogène et l'expression spécifique sous certaines conditions du gène correspondant. Il devient alors possible d'envisager toute une série d'expériences destinées à explorer le fonctionnement de différents systèmes intégrés - 


\section{REFERENCES}

15. Weaver $D$, Costantini $F$, Imanishi-Kari $T$, Baltimore D. A transgenic immunoglobulin $\mu$ gene prevents rearrangement of endogeneous genes. Cell 1985; 42: 1 1 7-27.

16. Le Meur M, Gerlinger P, Benoist C, Mathis D. Correcting an immune-response deficiency by creating $\mathrm{E} \propto$ transgenic mice. Nature 1985; 316: $3^{8-42}$

17. Yamamura $\mathrm{K}$, Kikutani $\mathrm{H}$, Folsom V, et al. Functional expression of a microinjected $\mathrm{E}_{\alpha}^{d}$ gene in $\mathrm{C}_{57} \mathrm{BL} / 6$ transgenic mice. Nature $1985 ; 316$ : 67-9.

18. Pinkert CA, Widera G, Cowing C, et al. Tissue-specific, inducible and functional expression of the $\mathrm{E}^{d} \mathrm{MHC}$ class II gene in transgenic mice. $E M B O$ I $1985 ; 4$ : 2225-30

19. Woychik RP, Stewart TA, Davis LG, D'Eustachio P, Leder P. An inherited limb deformity created by insertional mumgenesis in a transgenic mouse. Nature 1985; 318: 36-40.

20. Chisari FV, Pinkert LA, Milich DR, et al. A transgenic mouse model of the chronic hepatitis $B$ surface antigen carrier state. Science 1985, 230: $1157-60$.

21. Babinet $C$, Farza $H$, Morello D, Hadchouel M, Pourcel C. Specific expression of hepatitis B surface antigen (HBsAg) in transgenic mice. Science 1985; 230: $1160-3$.

22. Hammer RE, Pursel VG, Rexroad CE, et al. Production of transgenic rabbits sheep and pigs by microinjection. Nature 1985; 315: 680-3.

23. Kraemer D, Minhas B, Capehart J. Gene transfer into pronuclei of cattle and sheep zygotes. In: Costantini F, Jaenish R, ed. Genetic manipulation of the carly embryo. Banbury Report $n^{\circ}$ 20. New York: Cold Spring Harbor 1985: 221-7.

24. Townes TM, Lingrel JB, Chen HY, Brinster RL, Palmiter RD. Erythroid-specific expression of human $\beta$-globin genes in transgenic mice. $E M B O 7$ 1985; 4: 1715-23.

25. Shani M. Tissue-specific expression of ratmyosin light-chain 2 gene in transgenic mice. Nature 1985; 314: 283-6.

26. Krumlauf R, Hammer RE, Tilghman SM, Brinster RL. Developmental regulation of $\alpha$-Feto-protein genes in transgenic mice. Mol Cell Biol 1985; 5: 1639-48.

27. Magram J, Chada K, Costantini F. Developmental regulation of a cloned adult $\beta$-globin gene in transgenic mice. Nature 1985; 315: $33^{8-}$ 40.

28. Adams JM, Harris AW, Pinkert CA, et al. The c-myc oncogene driven by immunoglobulin enhancers induces lymphoid malignancy in transgenic mice. Nature 1985; 318 : 533-8.

29. Morello D, Moore G, Salmon AM, Yaniv $M$, Babinet $C$. Ubiquitous and regulated expression of an $\mathrm{H}-2 \mathrm{~K}$ /human growth hormone fusion gene in giant transgenic mice. EMBO 7 (sous presse).

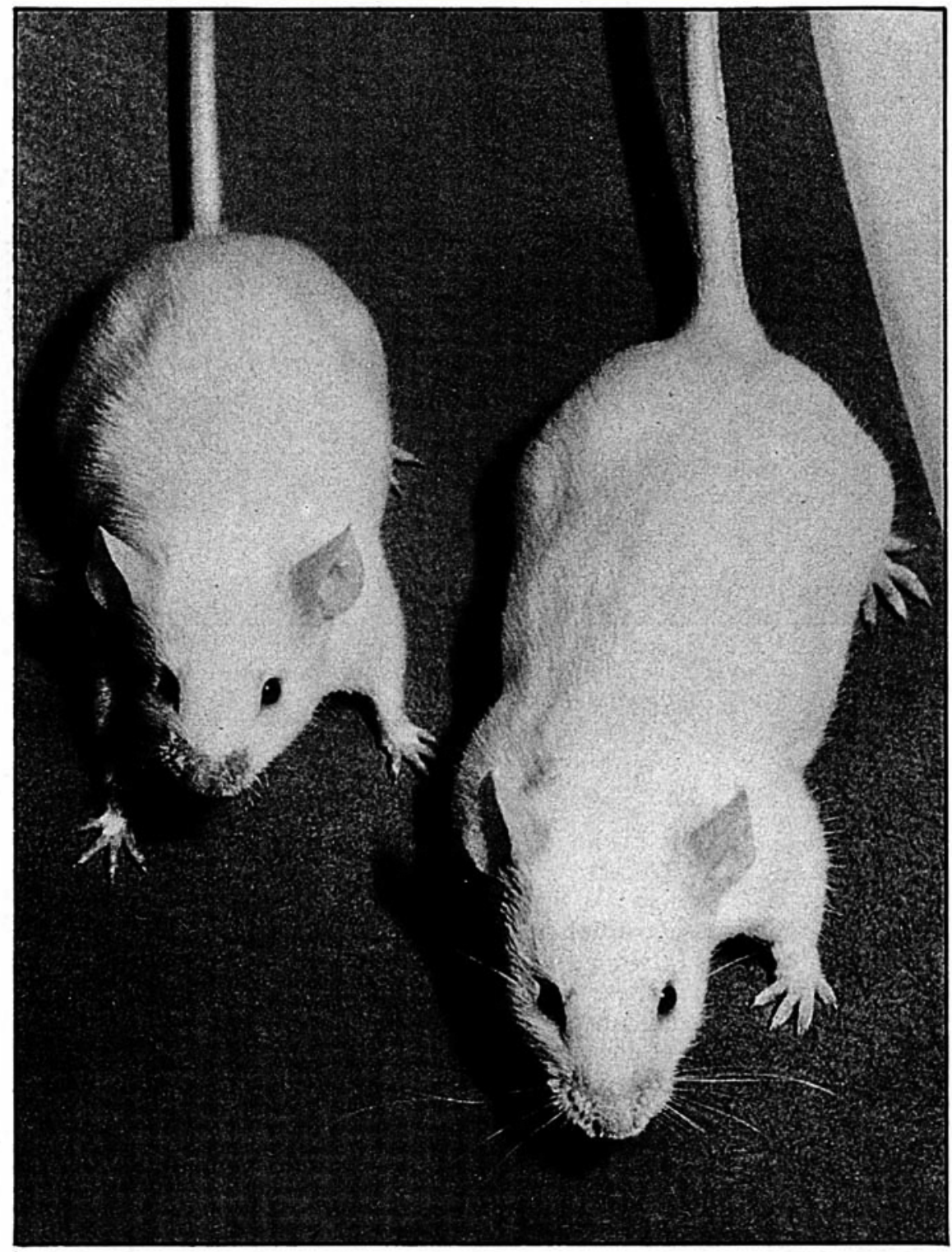

Figure 2. L'expression du gène de l'hormone de croissance dans une souris transgénique peut entraîner une croissance double (à droite) de celle d'une souris contrôle du même sexe et du même âge (à gauche).

par exemple le système immunitaire ou les différents réseaux de régulation hormonale - et le rôle fonctionnel des produits de gènes dont diverses observations suggèrent le rôle fondamental - par exemple les gènes oncogènes - et ceci dans le contexte de l'organisme considéré comme un tout.

\section{Les gènes hybrides}

Les techniques de la biologie moléculaire permettent presque à vo- lonté de "construire " des séquences d'ADN composites, c'està-dire faites de morceaux d'ADN de provenances différentes.

C'est ainsi que l'on peut obtenir des gènes comprenant les séquences régulatrices d'un gène $A$ (en particulier celles qui déterminent la spécificité du tissu) raboutées aux séquences codantes d'un gène $B$. En utilisant de telles constructions, on peut donc forcer l'expression du gène $B$ dans le ou les tissus où le gène $\mathrm{A}$ est normalement actif ( $t a$ - 


\begin{tabular}{|c|c|c|c|}
\hline UTILISATION & $\begin{array}{c}\text { Tableau II } \\
\text { E GÈNES HYBRIDES • DANS L }\end{array}$ & S SOURIS TRANSGÉNIQUES & \\
\hline gène & expression attendue dans : & expression observée dans : & références \\
\hline $\begin{array}{l}\text { insuline/antigène } T \text { du virus } \\
\text { SV40 } \\
\text { métallothionine souris/hormone } \\
\text { de croissance humaine } \\
\beta \text { globine souris/ } \beta \text { globine } \\
\text { homme } \\
\text { virus de la tumeur de la glande } \\
\text { mammaire de souris (MMTV)/ } \\
\text { proto-oncogène myc } \\
\text { activateur chaîne lourde lg/pro } \\
\text { to-oncogène myc } \\
\text { promoteur du gène d'histocom- } \\
\text { patibilité H-2K/hormone de } \\
\text { croissance humaine }\end{array}$ & $\begin{array}{l}\text { cellules } \beta \text { du pancréas } \\
\text { principalement le foie "*, le rein } \\
\text { et l'intestin et dans une moin- } \\
\text { dre mesure d'autres organes } \\
\text { lignée érythroïde } \\
\text { glande mammaire } \\
\text { lymphocytes B } \\
\text { tous les tissus à des degrés } \\
\text { variables }\end{array}$ & $\begin{array}{l}\text { id. } \\
\text { principalement le foie et dans } \\
\text { une moindre mesure, le cœur, } \\
\text { l'intestin et d'autres organes } \\
\text { id. } \\
\text { niveau faible } \\
\text { glande mammaire et autres tis- } \\
\text { sus suivant les souris } \\
\text { id. } \\
\text { id. }\end{array}$ & $\begin{array}{l}{[8]} \\
{[7]} \\
{[27]} \\
{[10]} \\
{[28]} \\
{[29]}\end{array}$ \\
\hline
\end{tabular}

- Les séquences injectées comprennent les séquences codantes d'un gène donné reliées aux séquences promotricesrégulatrices d'un autre gène. "* Après induction par le cadmium.

bleau II). En somme, par le biais d'une dérégulation artificielle de l'activité du gène $B$, on peut obtenir des lumières sur son rôle physiologique. Une illustration particulièrement frappante de cette stratégie a été fournie par Brinster, Palmiter et leurs collaborateurs [6]. Ces auteurs ont produit des souris transgéniques ayant intégré un gène hybride comprenant les séquences régulatrices du gène de la métallothionine *, liées à la partie codante du gène de l'hormone de croissance. Dans la plupart des cas, les souris issues des œufs ayant reçu cet ADN contenaient une grande quantité d'hormone de croissance dans leur sang et de plus devenaient "géantes", atteignant parfois plus de deux fois le poids de souris témoins n'ayant pas reçu le gène. L'analyse de ces souris a montré que l'expression du gène de l'hormone de croissance suivait dans une large mesure l'expression du gène métallothionine endogène c'est-à-dire

\footnotetext{
* Le gène de la métallothionine est normalement exprimé dans tous les organes, principalement le foie, les organes sexuels et le cerveau. Après induction par les métaux lourds, son expression a lieu de manière prédominante dans le foie puis le rein et lintestin et à un moindre degré dans les autres organes.

$m / s n^{\circ} 5$, vol. 2, mai 86
}

que les séquences régulatrices du gène exogène étaient fonctionnelles chez ces souris transgéniques : en particulier la synthèse d'hormone de croissance était stimulée par les métaux comme le cadmium ou le zinc, à l'instar de ce qui se passe pour le gène endogène. Au-delà du caractère spectaculaire de l'obtention de souris géantes, il faut souligner l'intérêt potentiel de ces animaux dans l'élucidation des mécanismes physiologiques qui interviennent dans la régulation de la croissance d'un organisme. Dans notre laboratoire, nous nous sommes intéressés au promoteur d'un gène d'histocompatibilité (le gène $\mathrm{H}-2 \mathrm{~K}$ ) qui fonctionne dans tous les tissus. Nous avons fusionné ce promoteur avec les séquences codantes du gène de l'hormone de croissance et obtenu des souris transgéniques qui produisent de grandes quantités de cette hormone dans leur sérum et dont la croissance est considérablement augmentée (figure 2). De plus, nous avons montré que l'expression du gène hybride dans les différents organes était proche de celle du gène $\mathrm{H}-2$ endogène correspondant. L'utilisation de promoteurs ubiquitaires, comme celui de la métallothionine ou des gènes d'histocompatibilité, permet donc de faire synthétiser dans tous les organes de l'animal le produit d'un gène, d'analyser l'effet produit et d'en tirer des enseignements intéressants sur son rôle.

\section{Les oncogènes}

La transformation d'une cellule normale en cellule maligne est un processus complexe et qui nécessite plusieurs étapes [7]. Le rôle joué par les oncogènes dans ce processus a été largement documenté ces dernières années. Les résultats récents obtenus avec des souris transgéniques pour différents oncogènes permettent de penser qu'elles sont également un outil de choix pour l'étude de la tumorigénèse. En utilisant une construction où le gène codant pour l'antigène $T$ (un oncogène du virus $\mathrm{SV}_{40}$, qui a notamment pour effet d'immortaliser les cellules qui le produisent) a été mis sous la dépendance du promoteur de l'insuline (promoteur normalement fonctionnel uniquement dans les cellules $\beta$ du pancréas) [8], des souris transgéniques qui meeurent toutes précocement de tumeurs du pancréas ont été obtenues. La recherche par immunofluorescence de l'antigène $T$ dans les îlots de Langerhans montre que seules les 
cellules $\beta$ en produisent (et non les cellules $\gamma$ ou $\delta$ ) : chez les souris très jeunes, les îlots sont normaux mais bientôt les cellules $\beta$ deviennent hyperplasiques puis certaines d'entre elles donnent naissance à des tumeurs. Ainsi, en "ciblant" la production de l'antigène $T$ à l'aide du promoteur de l'insuline, on démontre son rôle dans le processus de transformation tumorale. Le fait que toutes les cellules ne subissent pas cette transformation indique néanmoins que la production de l'antigène $T$ ne suffit pas à provoquer la formation de tumeurs.

Dans d'autres expériences, le gène codant pour l'antigène $T$ avec son propre promoteur (celui que l'on trouve dans le virus $S_{4}$ ) a été injecté dans l'œuf : les souris obtenues, qui ont intégré le gène, développent immanquablement des tumeurs du plexus choroïde et meurent vers l'âge de quatre mois [9]. Aussi bien les tumeurs que les lignées cellulaires établies à partir de celles-ci produisent l'antigène T. En revanche, les autres tissus de l'animal produisent peu ou pas d'antigène $T$. Il est donc probable que le promoteur naturel du gène $T$ se mette à fonctionner sous l'influence d'un facteur produit spécifiquement dans les cellules du plexus choroïde et que la synthèse d'antigène $T$ qui en résulte entraîne la formation d'une tumeur.

L'effet d'un autre oncogène, l'oncogène myc, a été examiné dans des expériences relevant de la même logique. Un gène composite a été construit qui comprend les séquences codantes de myc sous la dépendance de la région LTR (qui contient des séquences promotrices et des séquences activatrices) du virus de la tumeur mammaire de la souris [ro]. Des souris transgéniques obtenues avec cette construction ont développé des tumeurs de la glande mammaire après une ou deux gestations. Le gène exogène est exprimé dans différents organes, mais il faut l'induction hormonale liéc à la lactation pour que le niveau de production de myc soit suffisant pour donner naissance à une tumeur. Comme pour l'antigène $T$ dans le cas des tumeurs du pancréas, la surexpression de myc ne transformation tumorale puisque toutes les glandes mammaires de l'animal ne sont pas le siège de tumeurs.

Des expériences faites sur des cellules en culture ont permis de montrer que deux oncogènes agissent parfois de concert pour provoquer la transformation [7]. Les résultats obtenus avec les souris transgéniques pour les oncogènes $\mathrm{T}$ et myc indiquent clairement quelle direction peut être suivie pour élucider les rôles respectifs des différents oncogènes dans la naissance des tumeurs : en effet l'utilisation des gènes composites permet, comme nous l'avons vu, de cibler l'expression d'un oncogène. L'effet d'un oncogène donné dans différents tissus, ou de différents oncogènes dans un même tissu va donc pouvoir être examiné. De la même manière, il est envisageable de faire produire deux oncogènes par un même tissu et d'examiner ainsi leur coopération dans le processus de transformation tumorale.

\section{Le système \\ immunitaire}

Les différents problèmes que pose le fonctionnement du système immunitaire peuvent être abordés sous un jour nouveau, à l'aide des souris transgéniques. Des travaux antérieurs ont montré que le réarrangement des gènes d'immunoglobulines s'effectue spécifiquement dans les lymphocytes B (les immunoglobulines sont constituées de deux chaînes lourdes et de deux chaînes légères). Il en est de même dans les souris transgéniques ayant intégré ces gènes [I I-I5]. L'étude de ces souris a permis, en particulier, de montrer que l'expression par le gène exogène d'une molécule d'immunoglobuline entraînait la répression du réarrangement du gène endogène $[14,15]$.

Une telle observation apporte une explication au phénomène dit d'exclusion allelique qui se manifeste par le fait qu'un lymphocyte donné ne produit qu'un type de chaîne légère et un type de chaîne lourde correspondant au réarrangement d'un seul des deux allèles présents pour chacune des chaînes. Des souris transgéniques pour des gènes impliqués dans les interactions entre cellules du système immunitaire ont également été produites $[16,17,18]$. En particulier, des souris incapables de synthétiser une certaine classe de molécules du système d'histocompatibilité ont été "guéries" par l'introduction dans leur génome des gènes codant pour ces molécules.

\section{Mutagénèse par insertion}

Non seulement, comme nous l'avons vu plus haut, un gène étranger peut s'exprimer dans des souris transgéniques mais encore l'insertion de ce gène peut par elle-même avoir un effet sur le fonctionnement du génome des souris transgéniques obtenues. L'ADN étranger peut par exemple s'intégrer dans un gène important pour le développement et entraîner son inactivation. Lorsque, par des croisements appropriés, des souris homozygotes pour l'insertion auront été obtenues, l'inactivation totale du gène se manifestera par une anomalie du développement pouvant aller jusqu'à son arrêt complet (mutation léthale récessive). L'intérêt de ce type particulier de mutagénèse tient à ce que l'on dispose d'une sonde (l'ADN étranger) permettant de cloner le gène muté et donc, à terme, d'établir une relation directe entre le fonctionnement d'un gène donné et telle ou telle étape du développement. Plusieurs cas de ce type ont été décrits chez les souris transgéniques. En particulier, une équipe américaine a tout récemment décrit un cas d'anomalie de la formation des membres directement liée à une mutation induite par l'insertion d'une séquence d'ADN étranger [19]. La mutation a été localisée sur le chromosome 2. De plus, les auteurs ont montré que cette mutation était allélique avec deux autres mutations indépendantes apparues dans des élevages de laboratoire et entraînant le même phénotype. Les séquences adjacentes à l'insertion, représentant probablement le gène dont le fonctionnement a été perturbé, sont actuellement en cours de clonage. Ce gène va donc, selon toute vraisemblance, être identifié sous peu et le rôle de la (ou des) protéine(s) dont il gouverne la synthèse, étudié en relation avec 
la formation des membres.

Perspectives. $\mathrm{La}$ stratégie des souris transgéniques ouvre donc de larges voies pour l'étude des mécanismes moléculaires mis en œuvre au cours du développement : par la caractérisation de séquences d'ADN en cis des gènes et intervenant dans la régulation de leur expression dans le temps et dans l'espace; par la possibilité, grâce aux gènes hybrides, de "cibler" l'expression de différentes protéines dans différents tissus et d'en tirer des enseignements sur leur rôle; par la possibilité, grâce à l'effet mutagène éventuel de l'insertion, de découvrir les gènes impliqués dans les processus de morphogénèse. Mais d'autres perspectives se font jour : on peut envisager d'inactiver spécifiquement le fonctionnement de tel gène en produisant des animaux transgéniques ayant intégré ce gène sous une forme telle qu'il produise un messager "anti-sens" (celui-ci, en se combinant avec le messager normal va l'empêcher de fonctionner). Ceci devrait permettre par exemple de créer chez l'animal des modèles de maladies métaboliques. Dans le même ordre d'idée, on peut envisager d'introduire chez la souris le génome de tel ou tel virus humain et d'examiner les effets pathologiques de l'expression du génome viral. C'est le but d'expériences très récentes effectuées par une équipe de l'Institut Pasteur et une équipe américaine qui ont montré que l'on pouvait obtenir l'expression de l'antigène $\mathrm{HBs}$ du virus humain de l'hépatite $B$ chez des souris transgéniques ayant intégré une partie du génome du virus [20, 2r]. Enfin, si la souris est l'animal qui a fourni à ce jour le plus grand nombre d'animaux transgéniques, il faut noter l'intérêt d'étendre cette méthodologie à d'autres animaux, notamment des animaux d'élevage; en effet, des applications d'une importance pratique considérable peuvent être envisagées, comme par exemple une croissance accélérée ou l'obtention d'animaux résistants à des maladies. D'ores et déjà, des lapins, des cochons et des moutons transgéniques ont été obtenus [22] et des expériences sont en cours pour la production de bovins transgéniques $[23]$

\section{Summary}

DNA injected into the pronucleus of a fertilized egg may be integrated into the genome of the animal having developped from this egg. Such "transgenic animals" appear to be very useful for the study of various biological problems. To date the experiments using these animals have followed three main directions: (a) Analysis of the DNA sequences flanking the gene and involved in the control of its spatial and temporal expression. (b) Use of fused genes (containing the regulatory region of one gene fused to the coding sequences of another one) has permitted to target the expression of proteins to a given organ and to study the potential biological effect of this protein. (c) Integration of a foreign DNA into the genome of an animal may disrupt the functioning of an endogenous gene. In this way, new mutants of interest in the study of development, may be obtained. 